\title{
Generalized Trajectory Methods for Finding Multiple Extrema and Roots of Functions
}

\author{
C. M. YANG ${ }^{1}$ AND J. L. BECK ${ }^{2}$ \\ Communicated by F. E. Udwadia
}

\begin{abstract}
Two generalized trajectory methods are combined to provide a novel and powerful numerical procedure for systematically finding multiple local extrema of a multivariable objective function. This procedure can form part of a strategy for global optimization in which the greatest local maximum and least local minimum in the interior of a specified region are compared to the largest and smallest values of the objective function on the boundary of the region. The first trajectory method, a homotopy scheme, provides a globally convergent algorithm to find a stationary point of the objective function. The second trajectory method, a relaxation scheme, starts at one stationary point and systematically connects other stationary points in the specified region by a network of trjectories. It is noted that both generalized trajectory methods actually solve the stationarity conditions, and so they can also be used to find multiple roots of a set of nonlinear equations.
\end{abstract}

Key Words. Homotopy, relaxation, trajectory tracking, global optimization, roots, nonlinear equations.

\section{Introduction}

Many problems in engineering and science can be formulated as global optimization problems. In some applications, not only the global extrema but also all the local extrema are significant and need to be found. In any case, determination of these local extrema can be one step in a procedure to find the global extrema. Since the objective functions in many practical

'Graduate Student, Division of Engineering and Applied Sciences, California Institute of Technology, Pasadena, California.

${ }^{2}$ Professor, Division of Engineering and Applied Sciences, California Institute of Technology, Pasadena, California. 
applications are not convex, they may have multiple local and global extremal points in the region of interest. Finding all these multiple extrema is a challenging computational problem. Also, in contrast to local extrema, which can be characterized by the local behavior of the objective function, no practical and general criteria exist to determine whether a global extremum has been found.

A large number of publications have appeared during the past three decades on the subject of global optimization, presenting a variety of deterministic and stochastic methods (Refs. 1-2). A common feature of these global optimization methods is that some sort of global search strategy is incorporated into the algorithms. These strategies are essential due to the lack of local criteria for ascertaining global extrema, which may be located anywhere in the region of interest, and so they can be found only by systematically searching the region. The challenge is to perform this search in an efficient manner, since it is not computationally feasible to search exhaustively over a fine mesh unless the dimension of the parameter space is small. Most proposed global optimization methods use heuristics to improve the efficiency of the search and do not offer an absolute guarantee of finding the global extrema.

In this study, two generalized trajectory methods are combined to provide a novel and powerful approach for computation of multiple local extrema of an objective function. The first trajectory method is a homotopy one which provides a globally convergent algorithm to find a stationary point of the ojective function. The second trajectory method, called a relaxation scheme, starts at one stationary point and systematically connects other stationary points in a region by a network of trajectories. Both the homotopy and relaxation schemes actually solve the stationarity conditions, and so they can also be used to find multiple roots of a set of nonlinear equations.

\section{Generalized Trajectory Methods}

2.1. General Formulation and Assumptions. Let the objective function $f(x)$ be a sufficiently smooth scalar function of $n$ variables, with $x \in \mathscr{B} \subset R^{n}$, where $\mathscr{B}$ is an open region. We are interested in finding the points in $\mathscr{B}$ which give the greatest local maximum (GLM) and the least local minimum (LLM) of $f(x)$, if any. In many applications, prior knowledge of $f(x)$ and the choice of $\mathscr{B}$ can be used to conclude that the largest or smallest value of $f(x)$ on the closure $\overline{\mathscr{B}}$ of $\mathscr{B}$ is achieved in $\mathscr{B}$. In this case, the GLM or LLM of $f(x)$ in $\mathscr{B}$ gives the global maximum or minimum of $f(x)$ over $\overline{\mathscr{B}}$. In other cases, to find the global maximum over $\overline{\mathscr{B}}$, one strategy is to take the maximum of the GLM in $\mathscr{B}$ and the largest value of $f(x)$ on the boundary 
of $\mathscr{B}$. A similar strategy to find the global minimum over $\overline{\mathscr{B}}$ employs the LLM in $\mathscr{B}$. For these reasons, it is desirable to develop algorithms which compute the GLM and LLM of $f(x)$ in $\mathscr{B}$. Clearly, these points can be found if all the stationary points of $f(x)$ in $\mathscr{B}$ are located. It is assumed that the number of stationary points is finite so that they can each be checked for a local extremum.

It is well known that the stationary points of $f(x)$ satisfy the following $n$ stationarity conditions:

$$
\nabla f(x)=0 .
$$

Therefore, the problem of finding all the stationary points of $f(x)$ is reduced to the problem of finding all the roots of the set of nonlinear equations in (1). The basic idea of the generalized trajectory method is to embed (1) in a new set of equations,

$$
g(x, \lambda)=0, \quad \lambda \in R,
$$

where $g(x, \lambda) \in R^{n}$ and all the roots of (1) also satisfy (2) for some particular value of $\lambda$, say $\lambda=1$. Under some generic regularity assumptions on $g$, the solutions of (2) are one-dimensional manifolds or trajectories in $R^{n+1}$. Therefore, the roots of (1) can be found by tracking the trajectories defined by (2).

Two special generalized trajectory methods can be combined for finding the stationary points of $f(x)$. If stationary points of $f(x)$ exist in $\mathscr{B}$, but they are difficult to find using local optimization methods due to the lack of suitable initial guesses, a homotopy scheme can be used to find at least one stationary point. To find other stationary points, a relaxation scheme starting at a known stationary point can then be applied.

2.2. Homotopy Schemes. Several different names, such as embedding, invariant embedding, and parameter continuation, have been associated with homotopy schemes. The essence of a homotopy scheme is to smoothly transform a known solution to a simple problem into a solution of a target problem which is difficult to solve by embedding the simple and target problems in a more general problem. The function which describes the transition between the two problems is called a homotopy, and the stage of transformation is represented by a homotopy parameter. The simple solution evolves into the target solution by continuously varying the homotopy parameter (Refs. 3-4).

The major feature of homotopy schemes is global convergence, in the sense that they usually have a greatly expanded domain of convergence, so that it is possible to have solutions of the simple and target problems far apart and still have convergence. This property makes homotopy schemes 
drastically different from local iterative methods which need good initial guesses in order to converge. Therefore, homotopy schemes are particularly suitable for highly nonlinear problems for which good initial solution estimates are difficult to obtain.

Two main canonical homotopy schemes, Newton and fixed-point, have been proposed and studied in the literature, but only the latter is discussed here. The fixed-point homotopy is given by defining $g$ in (2) as

$$
g_{F}(x, \lambda)=(1-\lambda) M_{F}\left(x-x_{0}\right)+\lambda \nabla f(x)=0,
$$

where $x_{0}$ is a fixed arbitrary point in $\mathscr{B}$ and $M_{F}$ is a nonsingular $n \times n$ matrix. When $\lambda=0$, the simple problem

$$
g_{F}(x, 0)=M_{F}\left(x-x_{0}\right)=0
$$

has only one solution $x_{0}$, while (3) represents the original problem given in (1) when $\lambda=1$.

To apply this homotopy scheme, an aribitrary point $x_{0}$ in $\mathscr{B}$ is chosen and the homotopy trajectory defined by (3) is traced by allowing $\lambda$ to change from 0 . Whenever the homotopy trajectory reaches $\lambda=1$, a stationary point of $f(x)$ is found. It is noted that the homotopy trajectory may cross $\lambda=1$ several times. Therefore, it is sometimes possible to locate more than one stationary point by tracking a single homotopy trajectory. On the other hand, if there are no stationary points in $\mathscr{B}$, the homotopy trajectory will typically reach the boundary of $\mathscr{B}$ without crossing $\lambda=1$.

2.3. Relaxation Schemes. Suppose that at least one stationary point has been found by using homotopy schemes. To determine other stationary points in $\mathscr{B}$, if any, another type of generalized trajectory methods, which we call relaxation schemes, can be used. The basic idea of relaxation schemes is to have a special form of (2) which is as simple as possible and needs only the information of one stationary point. One simple relaxation scheme is given by defining $g$ in (2) as

$$
g_{R}(x, \lambda)=\nabla f(x)-(1-\lambda) v=0
$$

where $v$ is a given normalized constant vector in $R^{n}$. It is apparent that all the stationary points of $f(x)$ satisfy $g_{R}(x, 1)=0$. To apply the relaxation scheme, an aribtrary $v$ is chosen and the relaxation trajectory defined by (4) is traced from the known stationary point by allowing $\lambda$ to change from 1 . Whenever the relaxation trajectory crosses $\lambda=1$, another stationary point is found. Like homotopy trajectories, it is possible to locate more than one stationary point by tracking a single relaxation trajectory.

To increase the chance of finding all the stationary points, multiple relaxation schemes can be applied in a systematic way to set up a network 
of relaxation trajectories which hopefully connects all the stationary points. This proposed approach is systematic as opposed to other ad-hoc methods such as repeated application of a homotopy scheme with randomly selected $x_{0}$. One possibility is to use the standard orthonormal basis vectors $e_{1}$, $e_{2}, \ldots$, or $e_{n}$ as $v$. When $v$ is equal to $e_{i}$, the interpretation of (4) is that, in order to find other stationary points, the $i$ th constraint in (1) is relaxed while all the other constraints remain enforced. The vector $v$ can be systematically set to each basis vector in turn in order to relax a different constraint each time. In this case, $n$ relaxation trajectories are tracked from each stationary point that is found. This is the strategy followed in the numerical examples presented later. However, choices of $v$ other than the standard basis vectors could be explored and in some cases may find stationary points not found when $v$ is equal to $e_{i}$.

In fact, we have constructed an optimization problem in $R^{2}$ with multiple extrema and with a high degree of geometrical symmetry, for which the choices $v=e_{i}$ miss a centrally located extremum. Setting $v$ to any other choice of orthonormal basis vectors for $R^{2}$, however, leads to a network of relaxation trajectories which connects all the stationary points. It is not known whether it is possible to construct a problem in which the same extrema are missed for all choices of $v$.

2.4. Trajectory Tracking Algorithm. Both homotopy and relaxation trajectories need to be tracked by numerical algorithms, and therefore only a finite number of discrete points on a trajectory can be computed. To step from one point on a trajectory to an adjacent point on the same trajectory, some iterative scheme is required. The basic idea is to generate an initial guess for that adjacent point and then use some local iteration method to converge to the trajectory point. Different algorithms for trajectory tracking have been proposed (Refs. 4-7). These algorithms need to compute either the tangent vector of the trajectory or the Hessian matrix of the objective function, both of which are numerically expensive for complicated problems. To avoid such computations, a new and efficient trajectory tracking algorithm is presented and illustrated in Fig. 1.

Let $p=\left[x^{T}, \lambda\right]^{T}$, so (2) can be rewritten as

$$
g(p)=0, \quad p \in \mathscr{B} \times R .
$$

Suppose that $\Gamma$ is one component of the solution trajectory of $(5)$ and $p_{i}$ is the current point on $\Gamma$. To find an adjacent point $p_{i+1}$ on $\Gamma, p^{0}$ is used as an initial approximation. To render $p^{0}$, the secant direction which is determined by $p_{i-1}$ and $\mathrm{p}_{i}$ is used. Let $u$ be the unit vector in that secant direction; 


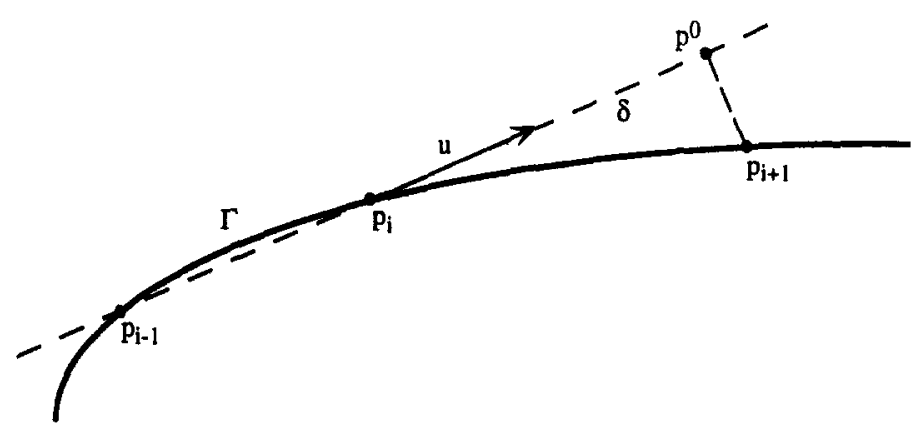

Fig. 1. Illustration of the trajectory tracking algorithm.

then, $p^{0}$ is chosen so that

$$
p^{0}=p_{i}+\delta u
$$

where $\delta$ is a given small number which controls the closeness of $p^{0}$.

Since $p_{i+1}$ is on $\Gamma$, it must satisfy (5). However, some additional constraint needs to be imposed in order to precisely determine the position of $p_{i+1}$. In the proposed algorithm, $p_{i+1}$ is chosen to be a root of the following set of equations:

$$
g(p)=0, \quad u \cdot\left(p-p_{i}\right)-\delta=0 .
$$

The second equation in (6) imposes a constraint on $p_{i+1}$ such that the projection of vector $p_{i+1}-p_{i}$ in the $u$ direction is equal to $\delta$. In other words, this constraint prescribes how far the step should be along $\Gamma$.

It is noted that (6) is just a set of $n+1$ nonlinear equations with $n+1$ variables. Given an initial guess $p^{0},(6)$ can be solved by using any local numerical iteration method for finding roots of a set of equations. If $\delta$ is small enough, the root $p_{i+1}$ will not be far away from the initial guess $p^{0}$ and convergence will occur. In the proposed algorithm, (6) is first transformed into a nonlinear least-squares optimization problem, which is then solved by either the Gauss-Newton or Levenberg-Marquardt method. These numerical algorithms are available in most general-purpose computational software packages.

A suitable choice of $\delta$ is essential to prevent divergence of the tracking algorithm and to minimize overstepping of a stationary point. Both the curvature of a trajectory and the accuracy requirement of tracking influence the selection of $\delta$. In practice, a suitably small default value of $\delta$ is chosen 
for a problem. If stepping from one point on a trajectory to an adjacent point on the same trajectory is not achieved in a certain number of local iterations, the default value of $\delta$ is halved and a new initial guess $p^{0}$ is rendered to restart the search for roots of (6). Once convergence has been achieved, $\delta$ is reset to its default value to find the next trajectory point.

\section{Properties of Homotopy and Relaxation Trajectories}

3.1. Differential Topology Aspects. The function $g(x, \lambda)$ in (2) can be viewed as a smooth map from $\mathscr{B} \times R$ to $R^{n}$. If $y^{*} \in R^{n}$ is a regular value, the pre-image theorem in differential topology asserts that the pre-images of $y^{*}$ are one-dimensional manifolds or trajectories in $\mathscr{B} \times R$. Therefore, the solutions of (2) will be smooth one-dimensional trajectories if $O \in R^{n}$ is a regular value of the map $g(x, \lambda)$. Fortunately, the famous Sard theorem (Ref. 8) asserts that almost all the points in $R^{n}$ are regular values, i.e., all the critical values in $R^{n}$ form a set of measure zero. Furthermore, the parameterized Sard theorem (Ref. 9) can be applied to the fixed-point homotopy scheme and the relaxation scheme to conclude that $0 \in R^{n}$ is a regular value for almost every $x_{0}$ in $\mathscr{B}$ and $v$ in $R^{n}$. Therefore, it is expected that the homotopy and relaxation trajectories are smooth and nonintersecting one-dimensional curves in $\mathscr{B} \times R$.

However, singularities, such as the kind of bifurcation in which two or more trajectories intersect at a single point, might occur along the homotopy and relaxation trajectories. Unfortunately, bifurcations cannot be completely treated by a simple analytic approach because of the huge variety of bifurcations (Refs. 10-11). Each type of bifurcation has its own distinct properties and needs to be classified and studied separately. There have been attempts to deal with some simple types of bifurcation using numerical procedures. One strategy is to derive conditions for the tangent directions of smooth trajectories intersecting at a simple bifurcation point (Ref. 12). Another approach is to perform an exhaustive search in the neighborhood of a bifurcation point for points on all the intersecting trajectories (Refs. 13-14).

In fact, multiple solutions can still be found without attacking the difficult bifurcation problem. The strategy used in this study is to ignore the occurrence of intersecting trajectories, if any. The fixed-point homotopy scheme is required to only find one stationary point. Then, multiple relaxation trajectories are systematically followed, as described earlier, to search for other stationary points instead of tracking bifurcating trajectories of a single homotopy or relaxation trajectory. Therefore, a treatment of bifurcation is not explicitly considered in the proposed global optimization algorithm. 
3.2. Trajectory Behavior. The Jacobian matrix of the fixed-point homotopy scheme defined in (3) is given by

$$
\nabla g_{F}(x, \lambda)=\left[(1-\lambda) M_{F}+\lambda \nabla \nabla f(x),-M_{F}\left(x-x_{0}\right)+\nabla f(x)\right] .
$$

At the starting point $p_{0}=\left[x_{0}^{T}, 0\right]^{T}$ on the homotopy trajectory, the Jacobian matrix is equal to

$$
\nabla g_{F}\left(x_{0}, 0\right)=\left[M_{F}, \nabla f\left(x_{0}\right)\right]
$$

Since the matrix $M_{F}$ is nonsingular, the Jacobian matrix in (7) has full rank. Therefore, $p_{0}$ is a regular point, and there is a unique and smooth onedimensional solution trajectory of (3) in the neighborhood of $p_{0}$ and passing through $p_{0}$. Assume that this trajectory segment is parameterized by the arc length $s$ in the neighborhood of $p_{0}$, i.e.,

$$
\begin{array}{ll}
x=x(s), & x(0)=x_{0}, \\
\lambda=\lambda(s), & \lambda(0)=0 ;
\end{array}
$$

then, (3) can be differentiated with respect to $s$ at $p_{0}$ and the result is

$$
M_{F} \dot{x}(0)+\dot{\lambda}(0) \nabla f\left(x_{0}\right)=0 .
$$

This leads to

$$
\left[\begin{array}{c}
\dot{x}(0) \\
\dot{\lambda}(0)
\end{array}\right] \propto\left[\begin{array}{c}
-M_{F}^{-1} \nabla f\left(x_{0}\right) \\
1
\end{array}\right] .
$$

Therefore, the unit tangent vector of the homotopy trajectory at the starting point $p_{0}$ can be computed from (8) and used as the starting value for $u$ in the trajectory tracking algorithm.

If the nonsingular matrix $M_{F}$ in (3) is symmetric, $g_{f}(x, \lambda)$ can be integrated with respect to $x$ so that

$$
\begin{aligned}
G_{F}(x, \lambda) & =\int_{x} g_{F}(x, \lambda) d x \\
& =(1-\lambda)(1 / 2)\left(x-x_{0}\right)^{T} M_{F}\left(x-x_{0}\right)+\lambda f(x) .
\end{aligned}
$$

It is clear from (9) that $G_{F}(x, \lambda)$ is made up of a simple quadratic function $(1 / 2)\left(x-x_{0}\right)^{T} M_{F}\left(x-x_{0}\right)$ and the objective function $f(x)$. If the leftmost $n \times n$ block of $\nabla g_{F}(x, \lambda)$ remains invertible along the trajectory, a local maximum of $f(x)$ will be reached if $M_{F}$ is negative-definite. Under the same condition on $\nabla g_{F}(x, \lambda)$, a local minimum of $f(x)$ will be reached if $M_{F}$ is positive-definite. Therefore, the fixed-point homotopy scheme can be biased toward either a local maximum or a local minimum for the first stationary point to be found. 
The Jacobian matrix of the relaxation scheme defined in (4) is given by

$$
\nabla g_{R}(x, \lambda)=[\nabla \nabla f(x), v] \text {. }
$$

If $x^{*}$ is a regular stationary point of $f(x)$, the Jacobian matrix at $p^{*}=$ $\left[x^{* T}, 1\right]^{T}$ is equal to

$$
\nabla g_{R}\left(x^{*}, 1\right)=\left[\nabla \nabla f\left(x^{*}\right), v\right] .
$$

Since the Hessian matrix $\nabla \nabla f\left(x^{*}\right)$ is nonsingular because $x^{*}$ is regular, the Jacobian matrix in (10) has full rank. Therefore, $p^{*}$ is a regular point and there is a unique and smooth one-dimensional solution trajectory of (4) in the neighborhood of $p^{*}$ and passing through $p^{*}$. Assume that this trajectory segment is parameterized by the arc length $s$ in the neighborhood of $p^{*}$, i.e.,

$$
\begin{array}{ll}
x=x(s), & x(0)=x^{*}, \\
\lambda=\lambda(s), & \lambda(0)=1 ;
\end{array}
$$

then, (4) can be differentiated with respect to $s$ at $p^{*}$ and the result is

$$
\nabla \nabla f\left(x^{*}\right) \dot{x}(0)+\dot{\lambda}(0) v=0 .
$$

This leads to

$$
\left[\begin{array}{c}
\dot{x}(0) \\
\dot{\lambda}(0)
\end{array}\right] \propto\left[\begin{array}{c}
-\left(\nabla \nabla f\left(x^{*}\right)\right)^{-1} v \\
1
\end{array}\right]
$$

Therefore, the unit tangent vector of the relaxation trajectory at the starting point $p^{*}$ can be computed from (11) and used as the starting value for $u$ in the trajectory tracking algorithm.

3.3. Multiple Trajectory Components. Under generic regularity assumptions, both homotopy and relaxation trajectories are smooth onedimensional curves in $R^{n+1}$. However, it is possible that the set of trajectories has more than one component. In that case, not all the stationary points lie on a single connected trajectory component. To find all the stationary points, all the separate components of the trajectory need to be found. There is no guaranteed complete solution for this task, and this remains as the major problem with the generalized trajectory methods.

Conditions which guarantee only one trajectory component were given in Ref. 15. But these conditions have only mathematical merit, since they are too strong and difficult to apply in most applications. Several methods have been proposed to deal with multiple trajectory components. One approach is to connect the separate trajectory components using certain one-dimensional curves which connect the trajectories through touching points (Ref. 16). However, not every trajectory component has a touching 
point. Another approach is try to connect different trajectory components at plus and minus infinity (Ref. 17). But, connection at infinity is not always possible because some trajectory components may be closed and remain bounded. Furthermore, reaching plus and minus infinity at the same point by different trajectory components is not guaranteed.

As discussed earlier, a new approach is presented here to deal with multiple trajectory components. Instead of using only one relaxation trajectory and dealing with the problem of connecting separate trajectory components, multiple relaxation trajectories are generated in a systematic way. The application of multiple relaxation generates redundancy and increases the chance of finding all the stationary points. Furthermore, the multiple trajectories passing through a stationary point can be traced simultaneously on parallel computers to increase efficiency in high-dimensional spaces.

\section{Numerical Examples}

4.1. Global Optimization Problem. In this example, the goal is to find all the points giving the global maximum of the objective function

$$
f(x)=\sum_{i=1}^{4} w_{i} G\left(x ; m_{i}, \Sigma_{i}\right), \quad x=\left[x_{1}, x_{2}\right]^{T} \in \mathscr{B} \subset R^{2},
$$

where

$$
\begin{aligned}
& w_{1}=w_{2}=1.0, \quad w_{3}=w_{4}=0.5, \\
& m_{1}=[2.5,2.5]^{T}=-m_{2}, \quad m_{3}=[2.0,-2.0]^{T}=-m_{4},
\end{aligned}
$$

and where

$G\left(x ; m_{i}, \Sigma_{i}\right)=\left[1 / 2 \pi \sqrt{\left|\Sigma_{i}\right|}\right] \exp \left[-(1 / 2)\left(x-m_{i}\right)^{T} \Sigma_{i}^{-1}\left(x-m_{i}\right)\right]$

is the two-dimensional Gaussian distribution function with mean $m_{i}$ and covariance matrix $\Sigma_{i}$. The covariance matrices $\Sigma_{i}$ are equal to $2 \times 2$ identity matrices, and the region of interest $\mathscr{B}$ is set to be $[-5,5] \times[-5,5]$. The three-dimensional mesh plot of $f(x)$ in (12) is given in Fig. 2. It is clear from the plot that $f(x)$ has nine stationary points in $\mathscr{B}$. Four of them give local maxima, one of them gives a local minimum, and the remaining four are saddle points. 


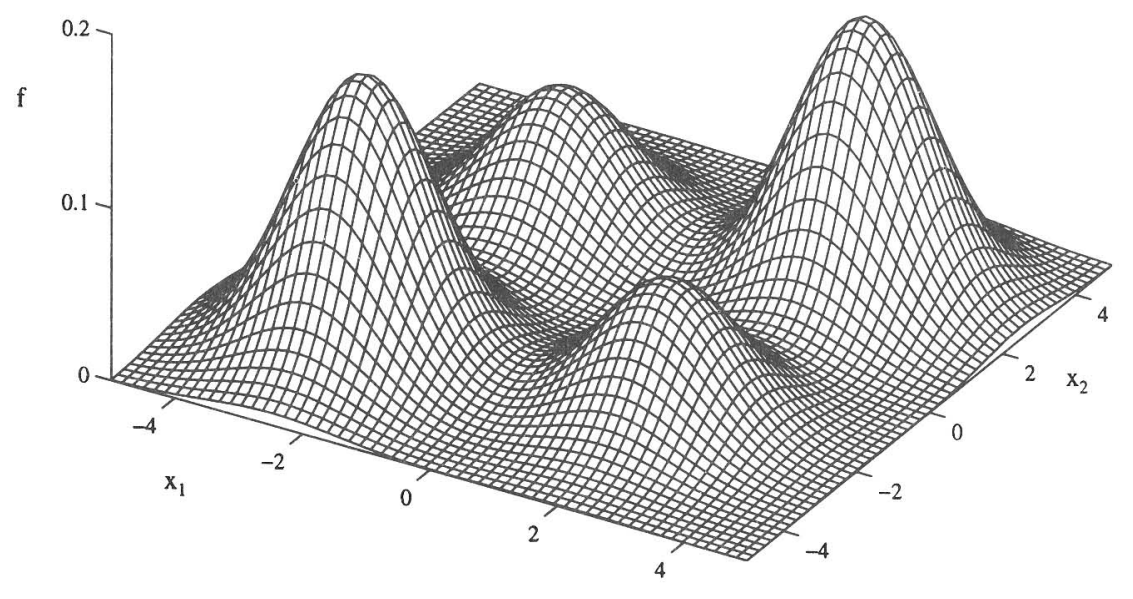

Fig. 2. Three-dimensional mesh plot of the objective function in (12).

The fixed-point homotopy scheme defined in (3) is first applied. The matrix $M_{F}$ is set equal to the negative of the $2 \times 2$ identity matrix, since we want the homotopy trajectory to converge to a local maximum. Three different initial guesses $\left[\mathrm{P}^{\mathrm{l}}(-1,0), \mathrm{P}_{2}(-4,1), \mathrm{P}_{3}(-2,4)\right]$ are used, and the homotopy trajectories are shown in Fig. 3 . It is noted that all three trajectories reach the same local maximum point $\mathrm{A}(-2,2)$.

From point $\mathrm{A}$, the relaxation scheme is applied to find the other stationary points. Two relaxation trajectories $\Gamma_{1}$ and $\Gamma_{2}$ going through $A$ are tracked and four more stationary points,

$\mathrm{B}(0.061,2.229), \quad \mathrm{C}(2.5,2.5), \quad \mathrm{D}(-2.229,-0.061), \quad \mathrm{E}(-2.5,-2.5)$

are located as shown in Fig. 4. By checking their Hessian matrices, points $B$ and $D$ are found to be saddle points while points $C$ and $E$ give local maxima. From point $B$, the relaxation trajectory $\Gamma_{3}$ is tracked and two more stationary points,

$$
F(0,0), \quad G(-0.061,-2.229),
$$

are found. Point $F$ gives a local minimum while point $G$ is a saddle point. By following the relaxation trajectory $\Gamma_{4}$ from point $C$, two more stationary points,

$$
\mathrm{H}(2.229,0.061), \quad \mathrm{I}(2,-2),
$$




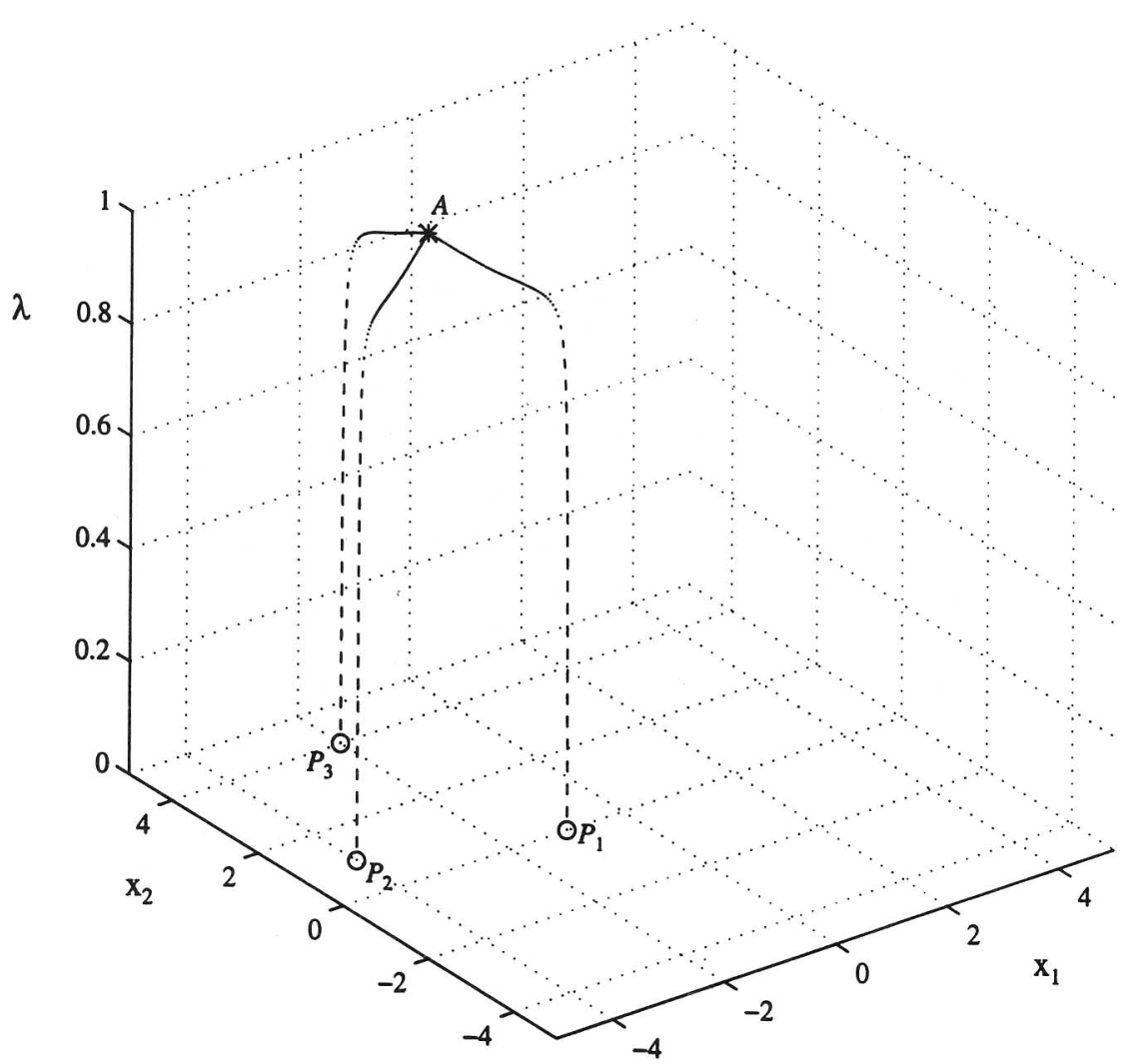

Fig. 3. Fixed-point homotopy trajectories for the objective function in (12).

are reached. Point $\mathrm{H}$ is a saddle point and point $\mathrm{I}$ gives a local maximum. It is noted that these stationary points can also be reached by tracking the relaxation trajectories $\Gamma_{5}$ and $\Gamma_{6}$.

All the special relaxation trajectories through the stationary points using $v$ equal to $e_{i}$ have now been explored so that the process is terminated. In this particular example, we can see from Fig. 2 that the nine points A to I are the only stationary points in $\mathscr{B}$. Therefore, it is concluded that all the stationary points in this example can be located using the presented algorithm. Furthermore, points $\mathrm{C}$ and $\mathrm{E}$ are local maxima with the same largest function value, so they give the greatest local maxima; in fact, they give the global maximum of the given $f(x)$ in $\mathscr{B}$ because the boundary values of $f(x)$ are all less than this value.

4.2. Model Identifiability Problem. In this example, a local model identifiability problem arising in statistical system identification (Ref. 18) is 


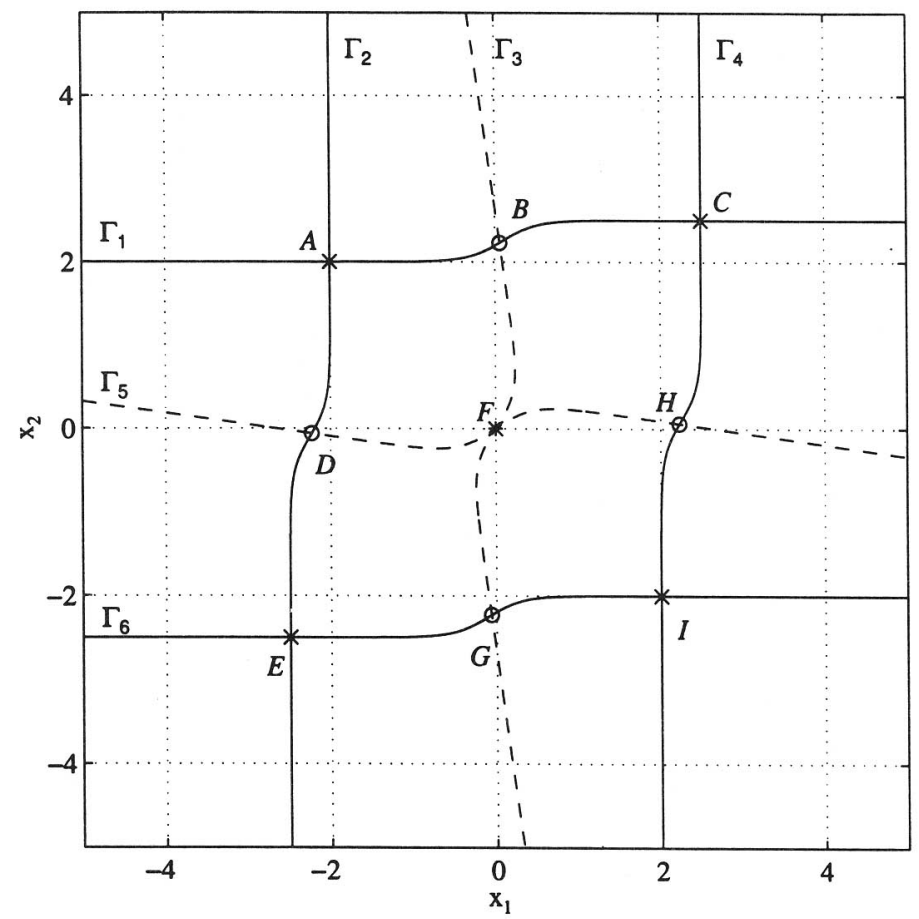

Fig. 4. Relaxation trajectories in the search of the global extrema of the objective function in (12).

studied by using the generalized trajectory methods. Consider a six-story building modeled using a set of linear chain models with equations of motion

$$
M \ddot{q}(t)+C \dot{q}(t)+K q(t)=f(t),
$$

where $M, C, K$ are the mass, damping, and stiffness matrices of the building; $f(t)$ is the exciting force acting on the building and $q(t)$ is the displacement vector at time $t$ whose components are the displacement of each floor of the building relative to its base. The mass matrix $M$ and stiffness matrix $K$ are given by

$$
M=m_{o}\left[\begin{array}{llll}
1 & & & 0 \\
& 1 & & \\
& & \ddots & \\
0 & & & 1
\end{array}\right] \text {, }
$$




$$
K=k_{o}\left[\begin{array}{ccccc}
\theta_{1}+\theta_{2} & -\theta_{2} & & & 0 \\
-\theta_{2} & \theta_{2}+\theta_{3} & -\theta_{3} & & \\
& -\theta_{3} & \ddots & & \\
& & & \theta_{5}+\theta_{6} & -\theta_{6} \\
0 & & & -\theta_{6} & \theta_{6}
\end{array}\right]
$$

where $m_{o}$ is the mass of each floor and $k_{o}$ is some nominal interstory stiffness. These two parameters are assumed to be known, whereas the stiffness parameters $\theta=\left[\theta_{1}, \theta_{2}, \ldots, \theta_{6}\right]^{T}$ are unknown. In the particular case considered here, the base acceleration $z(t)$ due to an earthquake is assumed to have been measured so that the exciting force $f(t)=-M 1 z(t)$ is known. The corresponding motion $q_{6}(t)$ at the roof is also assumed to have been measured. The problem is to determine the number of equivalent stiffness models consistent with these data.

Udwadia (Ref. 19) showed that the solution for $\theta$ is nonunique and that there is at most $6 !=720$ solutions. Beck and Katafygiotis (Ref. 18) have shown that there are 8 solutions if the data was generated by a uniform stiffness system with $\theta=[1,1,1,1,1,1]^{T}$. They showed that the solutions are actually given by those stiffness models which have the same natural frequencies as the actual system, i.e., all such models have the same roof response for a given base motion. Let $\omega_{1}, \omega_{2}, \ldots, \omega_{6}$ denote the six natural frequencies of a stiffness model, and let $\omega_{1}^{0}, \omega_{2}^{0}, \ldots, \omega_{6}^{0}$ be the corresponding natural frequencies of the uniform stiffness system. Therefore, to find all the equivalent stiffness models, all of the roots of the following set of nonlinear equations must be found:

$$
\omega_{1}(\theta)-\omega_{1}^{0}=0, \ldots, \omega_{6}(\theta)-\omega_{6}^{0}=0 .
$$

Since one of the roots of (14), i.e., the uniform stiffness model, is assumed to be known already, the relaxation scheme is applied to find all the other roots of (14). The stiffness parameters for the eight computed solutions are summarized in Table 1; they are the same as those presented in Ref. 18. Twelve relaxation trajectories, labeled as $\Gamma_{1}, \Gamma_{2}, \ldots, \Gamma_{12}$, were tracked in searching for these solutions. All the different stiffness models which can be reached by each trajectory are summarized in Table 2 . A • mark is placed in Table 2 to indicate that a particular stiffness model can be reached by a certain relaxation trajectory, while a blank means the opposite. Also, a $*$ mark is used to designate a relaxation trajectory which is a closed curve. For each trajectory, the relaxed natural frequency is indicated in the last column in Table 2. Also, it can be deduced from Table 2 that all of the 48 possible cases for relaxing each natural frequency at a time from 
Table 1. Equivalent stiffness models for a uniform six-degrees-offreedom linear chain model.

\begin{tabular}{ccccccc}
\hline No. & $\theta_{1}$ & $\theta_{2}$ & $\theta_{3}$ & $\theta_{4}$ & $\theta_{5}$ & $\theta_{6}$ \\
\hline 1 & 1.0000 & 1.0000 & 1.0000 & 1.0000 & 1.0000 & 1.0000 \\
2 & 1.5848 & 0.6963 & 1.2875 & 0.7574 & 1.1766 & 0.7898 \\
3 & 1.9970 & 0.7980 & 0.7095 & 1.3848 & 0.7113 & 0.8980 \\
4 & 2.0000 & 1.0000 & 1.0000 & 0.5000 & 1.0000 & 1.0000 \\
5 & 2.0932 & 1.0476 & 0.7240 & 0.7374 & 0.6705 & 1.2738 \\
6 & 2.2911 & 0.6304 & 0.9321 & 1.1774 & 0.9515 & 0.6631 \\
7 & 2.4913 & 0.8777 & 0.6514 & 1.1106 & 0.6672 & 0.9475 \\
8 & 2.8252 & 0.6753 & 0.8826 & 0.9021 & 0.8753 & 0.7520 \\
\hline
\end{tabular}

Table 2. Relaxation trajectories and reachable equivalent stiffness models.

\begin{tabular}{llllllllll}
\hline & \multicolumn{7}{c}{ Equivalent stiffness model } & Relaxed \\
\cline { 2 - 7 } Trajectory & 1 & 2 & 3 & 4 & 5 & 6 & 7 & 8 & $\omega$ \\
\hline${ }^{*} \Gamma_{1}$ & $\bullet$ & $\bullet$ & $\bullet$ & $\bullet$ & $\bullet$ & $\bullet$ & $\bullet$ & $\bullet$ & $\omega_{1}$ \\
${ }^{*} \Gamma_{2}$ & $\bullet$ & $\bullet$ & $\bullet$ & $\bullet$ & $\bullet$ & $\bullet$ & $\bullet$ & $\bullet$ & $\omega_{2}$ \\
${ }^{*} \Gamma_{3}$ & $\bullet$ & $\bullet$ & $\bullet$ & & & $\bullet$ & & & $\omega_{3}$ \\
${ }^{*} \Gamma_{4}$ & $\bullet$ & $\bullet$ & $\bullet$ & & & $\bullet$ & & & $\omega_{4}$ \\
${ }^{*} \Gamma_{5}$ & $\bullet$ & & $\bullet$ & & $\bullet$ & & $\bullet$ & & $\omega_{5}$ \\
${ }^{*} \Gamma_{6}$ & $\bullet$ & & & & $\bullet$ & & & & $\omega_{6}$ \\
${ }^{*} \Gamma_{7}$ & & $\bullet$ & & $\bullet$ & & $\bullet$ & & $\bullet$ & $\omega_{5}$ \\
${ } \Gamma_{8}$ & & $\bullet$ & & $\bullet$ & & & & & $\omega_{6}$ \\
${ }^{*} \Gamma_{9}$ & & & $\bullet$ & & & & $\bullet$ & & $\omega_{6}$ \\
${ }^{*} \Gamma_{10}$ & & & & $\bullet$ & $\bullet$ & & $\bullet$ & $\bullet$ & $\omega_{3}$ \\
${ }^{*} \Gamma_{11}$ & & & & $\bullet$ & $\bullet$ & & $\bullet$ & $\bullet$ & $\omega_{4}$ \\
${ } \Gamma_{12}$ & & & & & & $\bullet$ & & $\bullet$ & $\omega_{6}$ \\
\hline
\end{tabular}

each found stiffness solution has been covered by the 12 relaxation trajectories that were tracked.

\section{Conclusions}

It is not easy to claim that one global optimization method is more efficient than another method due to the lack of generally accepted measures for the performance of global optimization methods. Each method has its own advantages and drawbacks. It is our belief, however, that the combined homotopy and relaxation schemes provide a novel and powerful numerical procedure to find multiple local extrema as part of a strategy for global optimization. 


\section{References}

1. Arora, J. S., Elwakeil, O. A., Chahande, A. I., and Hsieh, C. C., Global Optimization Methods for Engineering Applications: A Review, Structural Optimization, Vol. 9, Nos. 3-4, pp. 137-159, 1995.

2. Törn, A., and Žrlinskas, A., Global Optimization, Lecture Notes in Computer Science, Springer Verlag, Berlin, Germany, Vol. 350, 1989.

3. Garcia, C. B., and Gould, F. J., Relations between Several Path-Following Algorithms and Local and Global Newton Methods, SIAM Review, Vol. 22, No. 3, pp. 263-274, 1980.

4. Keller, H. B., Lectures on Numerical Methods in Bifurcation Problems, Springer Verlag, Heidelberg, Germany, 1987.

5. Allgower, E., and Georg, K., Simplicial and Continuation Methods for Approximating Fixed Points and Solutions to Systems of Equations, SIAM Review, Vol. 22, No. 1, pp. 28-85, 1980.

6. Watson, L. T., Billups, S. C., and Morgan, A. P., Algorithm 652-HOMPACK: A Suite of Codes for Globally Convergent Homotopy Algorithms, ACM Transactions on Mathematical Software, Vol. 13, No. 3, pp. 281-310, 1987.

7. Desa, C., Irani, K. M., Ribbens, C. J., Watson, L. T., and Walker, H. F., Preconditioned Iterative Methods for Homotopy Curve Tracking, SIAM Journal on Scientific and Statistical Computing, Vol. 13, No. 1, pp. 30-46, 1992.

8. Guillemin, V., and Pollack, A., Differential Topology, Prentice-Hall, Englewood Cliffs, New Jersey, 1974.

9. Chow, S. N., Mallet-Paret, J., and Yorke, J. A., Finding Zeros of Maps: Homotopy Methods That Are Constructive with Probability One, Mathematics of Computation, Vol. 32, pp. 887-899, 1978.

10. KuZnetsov, Y. A., Elements of Applied Bifurcation Theory, Springer Verlag, New York, New York, 1995.

11. Golubitsky, M., and SchaefFer, D. G., Singularities and Groups in Bifurcation Theory, Vol. 1, Springer Verlag, New York, New York, 1985.

12. Doedel, E., Keller, H. B., and Kernevez, J. P., Numerical Analysis and Control of Bifurcation Problems, I: Bifurcation in Finite Dimensions, International Journal of Bifurcation and Chaos, Vol. 1, No. 3, pp. 493-520, 1991.

13. Kearfott, R. B., Some General Bifurcation Techniques, SIAM Journal on Scientific and Statistical Computing, Vol. 4, No. 1, pp. 52-68, 1983.

14. FujI, F., and Choong, K. K., Branch Switching in Bifurcation of Structures, ASCE Journal of Engineering Mechanics, Vol. 118, No. 8, pp. 1578-1596, 1992.

15. Diener, I., On the Global Convergence of Path-Following Methods to Determine All Solutions to a System of Nonlinear Equations, Mathematical Programming, Vol. 39, No. 2, pp. 181-188, 1987.

16. Diener, I., and Schaback, R., An Extended Continuous Newton Method, Journal of Optimization Theory and Applications, Vol. 67, No. 1, pp. 57-77, 1990.

17. Seader, J. D., Kuno, M., Lin, W. J., Johnson, S. A., Unsworth, K., and WISkIN, J. W., Mapped Continuation Methods for Computing All Solutions to General Systems of Nonlinear Equations, Computers and Chemical Engineering, Vol. 14, No. 1, pp. 71-85, 1990. 
18. BeCK, J. L., and KATAFYGIOTIS, L. S., Updating of a Model and Its Uncertainties Utilizing Dynamic Test Data, Proceedings of the 1st International Conference on Computational Stochastic Mechanics, Computational Mechanics Publications, Boston, Massachusetts, pp. 125-136, 1991.

19. Udwadia, F. E., Some Uniqueness Results Related to Soil and Building Structural Identification, SIAM Journal on Applied Mathematics, Vol. 45, No. 4, pp. 674$685,1985$. 